Applied Spectroscopy

\title{
Broadband and High-Resolution Static Fourier Transform Spectrometer with Bandpass Sampling
}

\author{
Meriç Özcan ${ }^{*}$ and Behzad Sardari*
}

\begin{abstract}
In this study, experimental demonstration of a static Fourier transform spectrometer (static-FTS), based on division of the spectrum into multiple narrowband signals, is presented. The bandpass sampling technique used in this novel spectrometer solves the Nyquist sampling rate limitations and enables recording of wideband spectrum in high resolution. The proposed spectrometer not only has the potential of operating in a wide spectral range, but also has a resolution potential better than $2 \mathrm{~cm}^{-1}$.
\end{abstract}

\section{Keywords}

Static Fourier transform spectrometer, bandpass sampling, broadband spectrometer, high-resolution spectrometer

Date received: II September 2017; accepted: 12 February 2018

\section{Introduction}

Broadband, high signal-to-noise $(\mathrm{S} / \mathrm{N})$ ratio, and highresolution spectrometers are highly in demand for industrial applications, environmental measurements, and for scientific measurements. ${ }^{I-5}$ The spectrometers can be classified essentially in two categories: dispersive spectrometers and Fourier transform spectrometers (FTS). Dispersive spectrometers rely on the diffraction of light from the diffraction gratings (DG), while the FTS relies on the self-interference of light through an interferometer with a variable path-length difference (PLD); the most common interferometer used for this purpose is a Michelson interferometer. ${ }^{6-8}$ The PLD for light interference through a Michelson interferometer is generally created in two different ways: via a moving system or a static mechanism (static-FTS). In a moving system, one of the mirrors is fixed in space while the second one is translated to produce the PLD. Different static-FTSs based on different interferometer configurations, such as the static Michelson interferometer with one flat mirror and one staircase mirror in which the individual stairs create a range of PLD, 9,10 MachZehnder or Michelson interferometers that use an array of step gratings with each grating having a different step height, "I spatially modulated prism, ${ }^{12}$ etc. are already proposed and implemented.

The FTSs, in general, have some advantages over dispersive spectrometers such as the multiplex (Fellgett's advantage), the high throughput (Jacquinot's advantage), and the wavelength accuracy (Connes' advantage). ${ }^{8,13}$ In FTSs with a moving mechanism, a very high resolution and precise distance sampling is required to satisfy the Nyquist sampling rate, which is a complicated and costly process. Hence, static mechanisms are developed to overcome this drawback of the moving mechanism-based FTSs. However, the existing static-FTSs have to operate either at longer wavelengths or in a narrowband spectrum, and in general they have low resolution. ${ }^{9,14}$ Also, fabrication of staircase mirrors in nano or micro scales is costly and requires high-tech facilities.

Here we demonstrate for the first time the operation of a potentially broadband, high-resolution, and low-cost static-FTS that uses a bandpass sampling technique as discussed earlier. ${ }^{15,16}$ Because the sampling rate of a bandpass signal depends on the signal bandwidth and the central frequency rather than the highest frequency, it can be sampled with a lower frequency rather than twice the highest frequency (required otherwise according to Nyquist sampling theorem), which in the FTS concept means larger sampling steps for the moving mirror ${ }^{17}$ are acceptable. This is a

Faculty of Engineering and Natural Sciences, Sabanci University, İstanbul, Turkey

${ }^{\dagger}$ Equal contributors.

Corresponding author:

Meriç Özcan, Sabanci Universitesi, Electronics Engineering, Tuzla 34956, Turkey.

Email: meric@sabanciuniv.edu 
definite advantage since there is no need to have micro- or nano-size steps on the staircase mirror. Sampling and recovery of a bandpass signal is summarized in Fig. I. Here the sampling frequency is lower than the Nyquist rate, and the recovery of the original signal is accomplished by a numerical translation of the bandpass filtered sampled signal to its original frequency. If the highest frequency of the signal is equal to an integer multiple of the bandwidth of the signal, then sampling with twice the bandwidth will produce perfectly inter-woven shifted spectral replicas. In this example, we show a general case where, depending on the sampling frequency, the lowest shifted spectral replica may have the correct spectral orientation or the flipped spectral orientation. Therefore, in order to recover the original signal after sampling we have to calculate two sets of frequencies:

$$
\begin{aligned}
& f_{b}=f_{L}-M f_{s} \\
& f_{b}^{\prime}=-f_{H}+(M+1) f_{s}
\end{aligned}
$$

where $M$ is the nearest integer $\leq M=f_{L} / f_{S}$ and $f_{s}$ is the sampling frequency. Then, the smaller of $f_{b}$ and $f_{b}^{\prime}$ determine the starting frequency of the bandpass filter that recovers the original signal. If $f_{b}^{\prime}$ is smaller, the recovered spectrum has to be flipped numerically. Frequency is the common variable for electrical signals but here we deal with optical signals; therefore, it is more appropriate to use the wavelength. Hence, for a bandpass optical signal, sampling step $\delta$ does not have to obey Nyquist sample rate limitation but can be set in a range as: ${ }^{15}$

$$
\frac{(m-1)}{2} \lambda_{L} \leq \delta \leq \frac{m}{2} \lambda_{S}, \quad m \leq \frac{\lambda_{L}}{\lambda_{L}-\lambda_{S}}
$$
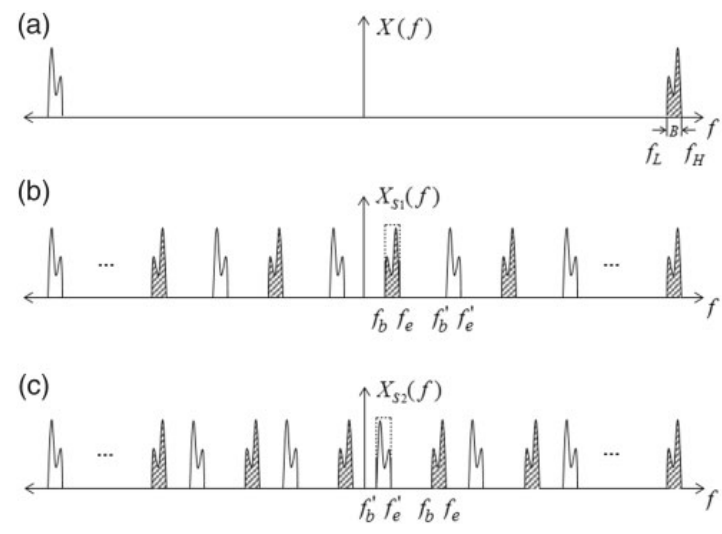

Figure I. (a) A bandpass signal $X(f)$ with bandwidth $B$. Sampled signal spectrum is given as $X_{s}(f)=\sum X\left(f \pm N f_{s}\right)$, and depending on the sampling rate, the lowest shifted spectral replica is exactly the same orientation (b) or the flipped version of the original spectrum (c). where $\lambda_{S}$ and $\lambda_{L}$ are the shortest and the longest wavelengths in the signal spectrum, respectively, and $m$ is defined as any integer number $\leq \lambda_{L} /\left(\lambda_{L}-\lambda_{S}\right)$. Therefore, if a broadband signal is divided into multiple narrowband channels, and if a sampling step $\delta$ can be found that is common for all the channels, one can use a static-FTS with staircase mirrors with that common sampling step size.

\section{Experimental Set-Up}

Experimental configuration is composed of two integral parts: the first one is a double diffraction grating structure to disperse the input spectrum in a horizontal direction so that each column of the charge-coupled device (CCD) will receive a narrow band of the spectrum, and the second one is a static Michelson interferometer to make different PLDs in the vertical direction, as illustrated in Fig. 2. After the pinhole in the input section, light is reflected from a curved mirror to a pair of gratings. First, diffraction grating receives the light as a vertical strip and disperses horizontally as a function of wavelength and the second diffraction grating collimates this beam in the horizontal (y direction). For a double diffraction grating structure, as shown in Fig. 3, the relevant equations for the diffraction angles at wavelength $\lambda_{n}$ are as follows:

$$
\begin{aligned}
& D G_{1}: p \lambda_{n}=d\left(\sin \alpha_{i n}+\sin \beta_{n}\right) \\
& D G_{2}: p \lambda_{n}=d\left(\sin \alpha_{n}+\sin \gamma\right)
\end{aligned}
$$

where $d$ is the diffraction grating period, $p$ is the order of diffraction, and angles are as indicated on Fig. 3. If the $D$ is the distance between the grating pair and the CCD pixel dimension is $P$, one can write $L=P / \cos (\gamma)$ and $L=D \times\left[\tan \beta_{n+1}-\tan \beta_{n}\right]$. Then, we can simply calculate the wavelength $\lambda_{n+1}$ that falls on the adjacent pixel:

$$
\lambda_{n+1}=d \times\left[\sin \alpha_{i n}+\sin U\right]
$$

where $U=\tan ^{-1}\left(\tan \beta_{n}+P / D \cos \gamma\right)$. Therefore, ignoring the inter-pixel spacing, the spectral range per pixel is $\left(\lambda_{n+1}-\lambda_{n}\right)$.

The second part of the spectrometer, which is composed of a beam-splitter (BS), a flat mirror, and a staircase mirror (in actual set-up, a diffraction grating in Littrow configuration is used), works as follows: the collimated beams at the output of the double diffraction grating structure enter the BS, then split into two different arms; one arm is reflected from the flat mirror and the other one is reflected from the stair-case mirror. The reflected beams from these mirrors interfere on a CCD camera which is placed on the back of the BS, as depicted in Fig. 2. The reflected light experiences different PLDs due to the staircase mirror which is realized by a diffraction grating in 
(a)

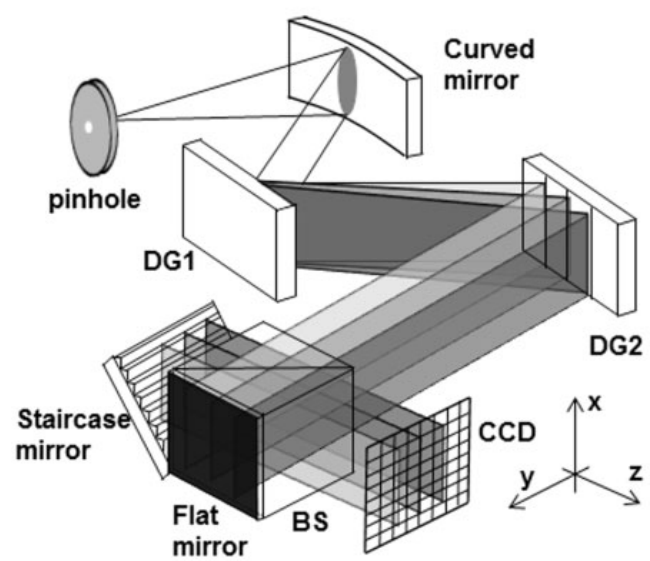

(b)

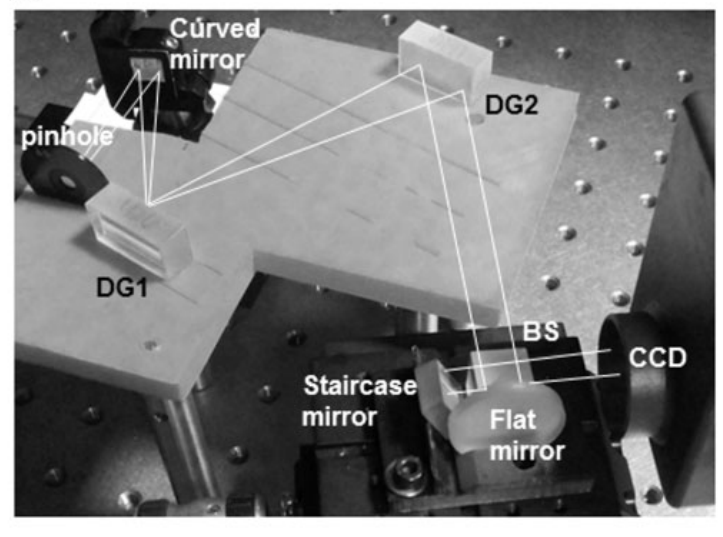

Figure 2. (a) A drawing of the proposed static-FTS. At the entrance, a $5 \mu \mathrm{m}$ pinhole before a concave mirror of $50 \mathrm{~mm}$ focal length is used. Two diffraction gratings (DG) of 600 grooves $/ \mathrm{mm}$ spreads the beam in wavelength in horizontal direction before a Michelson interferometer composed of a 0.5 in. cube BS, a flat mirror, and a diffraction grating with 1200 grooves $/ \mathrm{mm}$. This diffraction grating is used in Littrow configuration to realize the staircase mirror. (b) An actual photo of the experimental set-up.

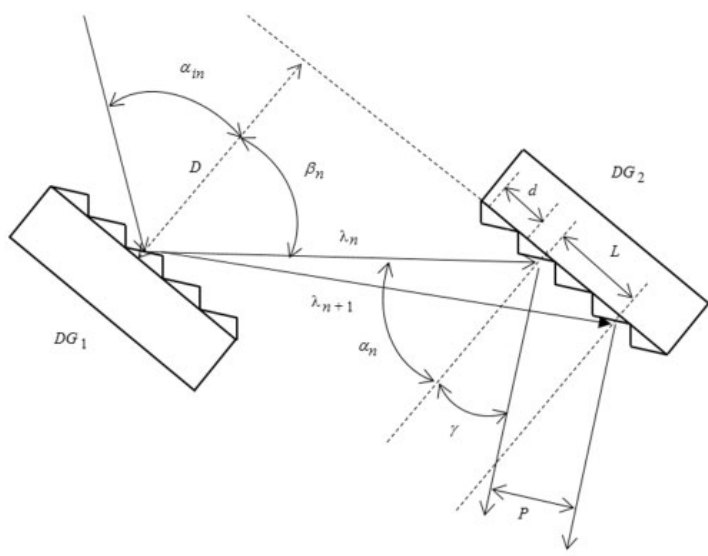

Figure 3. Double diffraction grating structure, in which two similar diffraction gratings are placed parallel to each other to disperse the beam in horizontal direction.

Littrow configuration. On the CCD, the $x$-direction is allocated for PLD variation and the $y$-direction is allocated for spectral segments (bandpass signals). In order to obtain the spectrum of a segment (call it $I(y)$ ), we must calculate the Fourier transform on the pixels of the yth column on the CCD. When the interferometer is configured with a flat mirror and a staircase mirror, the total electric field on the $m$ th pixel of a segment would be $\vec{E}=\vec{E}(k) \cos (\omega t)+$ $\vec{E}(k) \cos (\omega t+2 m k \delta)$, where $k$ is the wavenumber and $\delta$ is the step size, as illustrated in Fig. 4. Then, the time averaged recorded intensity $I$ with a distribution of wavelengths is given as:

$$
I=\left\langle\vec{E} \cdot \vec{E}^{*}\right\rangle=\int A(k)[1+\cos (2 m k \delta)] \mathrm{d} k
$$

where $A(k)$ is the spectrum of interest. However, here we have used a diffraction grating in Littrow configuration to realize the staircase mirror since it solves the fabrication issues of the micro staircase mirrors, and it is possible to use off-the-shelf diffraction gratings. This configuration is equivalent to a mirror with $N$ sub-steps per pixel, as shown in Fig. 4. For a staircase mirror with $N$ sub-steps and a single wavelength, the electric field $(\vec{E})$ on the $m$ th pixel of the CCD can be calculated as:

$$
\vec{E}=N \vec{E}(k) \cos (\omega t)+\sum_{i=1}^{N} \vec{E}(k) \cos (\omega t+2 m k \delta+2 i k \Delta)
$$

In the experiments, we used a diffraction grating with $1200 \mathrm{~g} / \mathrm{mm}$ and blaze angle of $26.4^{\circ}$, which the total $N=7$ grooves match a pixel width of the CCD. Therefore, the interferogram intensity in this case, after some algebra, is given as:

$$
I(\delta)=\int A(k)\left[\frac{49}{2}+2 S^{2}+14 S \cos (2 m k \delta+8 k \Delta)\right] \mathrm{d} k
$$

where $A(k)$ is the amplitude of the signal with wavenumber $k$ and $S=0.5+\cos (2 k \Delta)+\cos (4 k \Delta)+\cos (6 k \Delta), \delta$ is the staircase mirror depth, and $\Delta$ is the depth of diffraction grating grooves, as shown in Fig. 4. In order to calculate the $A(k)$, we calculate the Fourier transform of the recorded intensity I, then divide it by I4S. An important note here is that in the ideal case where the interferogram intensity is calculated with Eq. 6, 50\% of the input signal appears as a DC signal, while in our case here (Eq. 8), the DC value is 
(a)

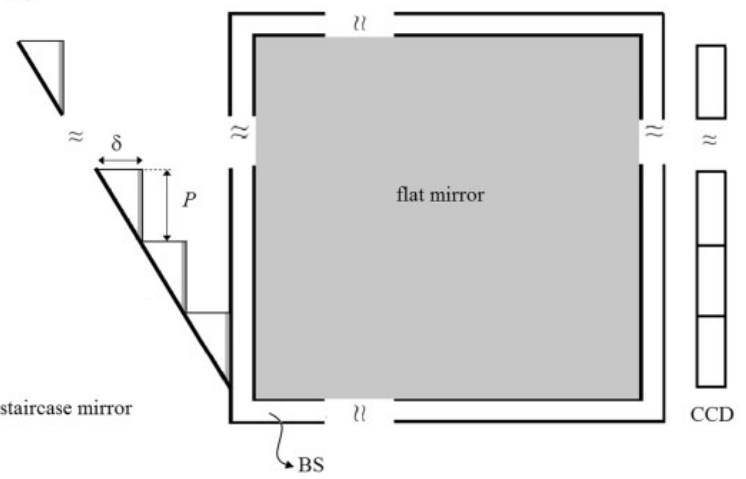

(b)

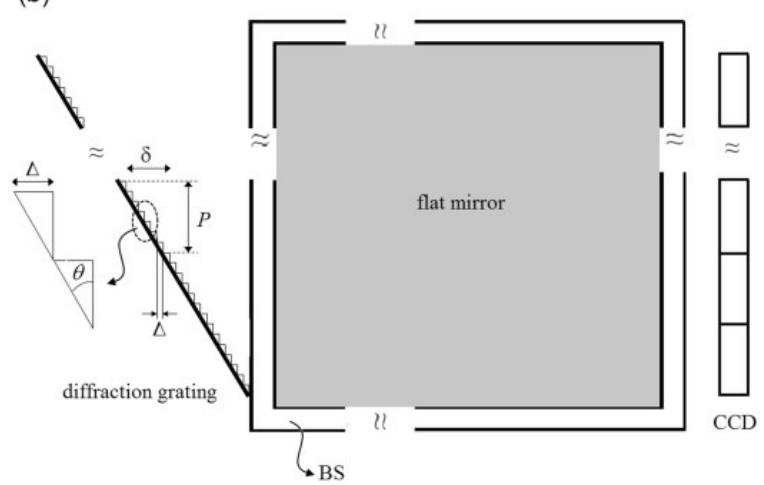

Figure 4. Side view of the interferometer from the flat mirror side. (a) Ideal experimental configuration. (b) A diffraction grating with 1200 grooves $/ \mathrm{mm}$ is used in Littrow configuration to realize staircase mirror with $\theta=26.4^{\circ}$ and $\delta=7 \Delta=2.6 \mu \mathrm{m}$.

about $75 \%$. This means reduction in signal contrast or visibility in our actual experimental configuration.

For the proof of principle experiments, we recorded $140 \mathrm{~nm}$ wide spectrum of signals in the range of 525$665 \mathrm{~nm}$ with a $1280 \times 1024$ pixel CCD camera. The pixel dimension of $5.25 \mu \mathrm{m}$ is a common acceptable sampling step for these 1280 narrowband signals (calculated with Eq. 2) except the three segments. For this particular example, the segments 253, 534, and 848 violate the BPS theorem; therefore, for those segments the resolution will be limited to the resolution of the dispersive section/dimension. It is important to note here again that when this spectrum range has to be recorded with a traditional static-FTS, the sampling steps must be $<262 \mathrm{~nm}$ (stair depth of $132 \mathrm{~nm}$ ) due to Nyquist sampling theorem.

\section{Results and Discussion}

The unique configuration of our proposed spectrometer actually allows the resolution and bandwidth selection independently. The delay part determines the pixel resolution and the number of segments determine the overall bandwidth of the spectrometer. The device bandwidth is limited by the CCD array width for a given dispersion from the grating pair, here in our case the width of $6.65 \mathrm{~mm}$ provides $140 \mathrm{~nm}$ bandwidth. The maximum delay is $2 N_{x} \delta$ where $N_{x}$ is the number of CCD pixels in the delay direction (vertical direction in here) and $\delta$ is the depth of staircases as shown in Fig. 4. Therefore, the pixel resolution is limited to the inverse of this delay, and for the set-up presented $(1 / 5.32 \mathrm{~mm})=1.9 \mathrm{~cm}^{-1}$ is the pixel resolution.

The recorded spectrums of a halogen light source and a light emitting diode (LED) with the proposed spectrometer are shown in Fig. 5 below. The black solid lines indicate the recorded spectrum with our device and the dashed lines indicates the spectrum recorded with OceanOptics' HR4000 spectrometer. There is a good agreement with the recorded spectrums of the two, the slight discrepancy of the recorded spectrums is currently under investigation.
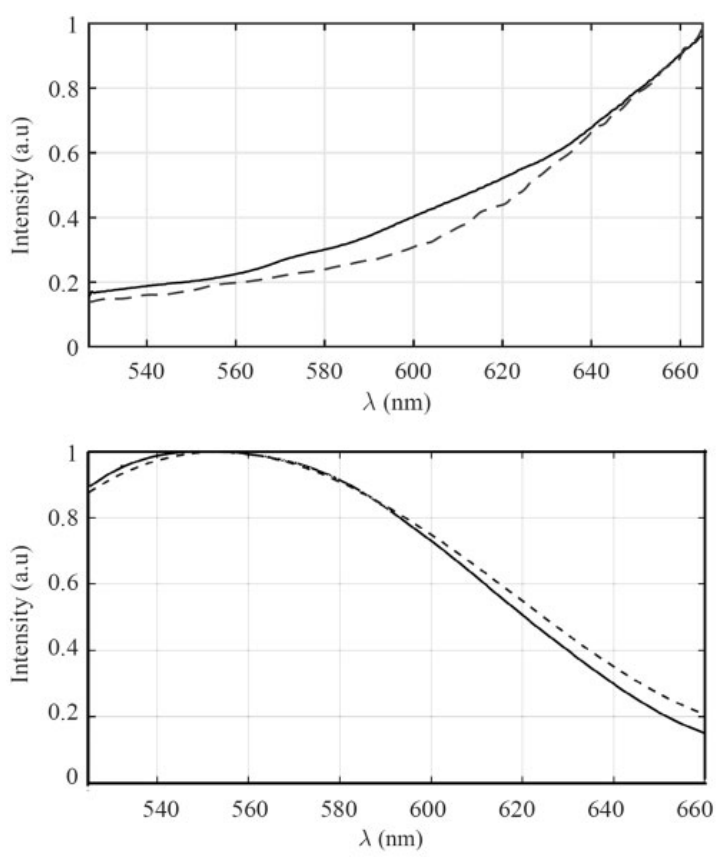

Figure 5. Top: Spectrum of Halogen light source. Bottom: Spectrum of a LED. Solid lines indicate recordings of the proposed static-FTS and dashed lines indicate recordings of the OceanOptics QEPro spectrometer. The resolution for the staticFTS is roughly $1.9 \mathrm{~cm}^{-1}$. Total spectrum recording time is $5 \mathrm{~s}$.

In another experiment, Fig. 6 shows the recorded CCD images when green and red $\mathrm{He}-\mathrm{Ne}$ lasers were both present in the input. The calculated spectrum is shown in Fig. 7, where the peaks are at $532 \mathrm{~nm}$ for the green laser and $632.8 \mathrm{~nm}$ for the red laser.

In conclusion, a compact, high-resolution and potentially broadband static-FTS based on bandpass sampling is demonstrated. The experimental spectrometer functions as a hybrid dispersive and a static FT spectrometer and has $140 \mathrm{~nm}$ bandwidth with $1.9 \mathrm{~cm}^{-1}$ resolution. If we consider only the dispersive part, the pixel resolution would 


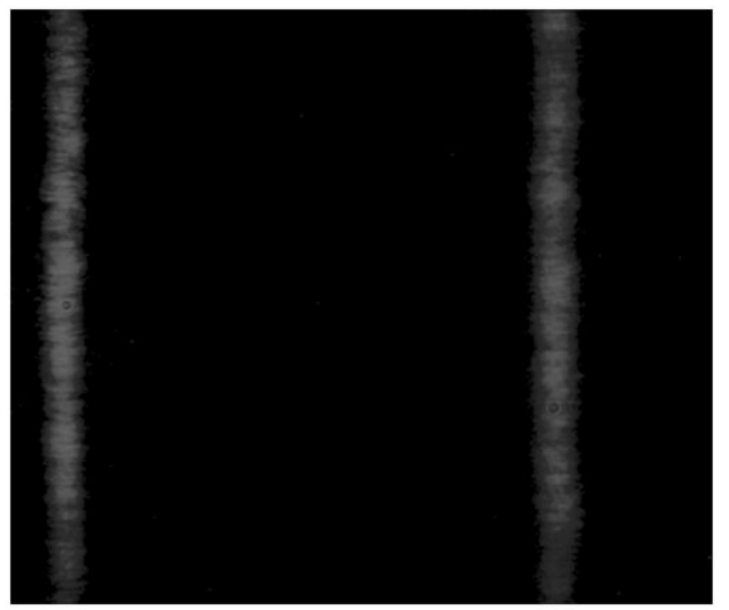

Figure 6. Recorded image of one green and one red $\mathrm{He}-\mathrm{Ne}$ laser by the CCD.

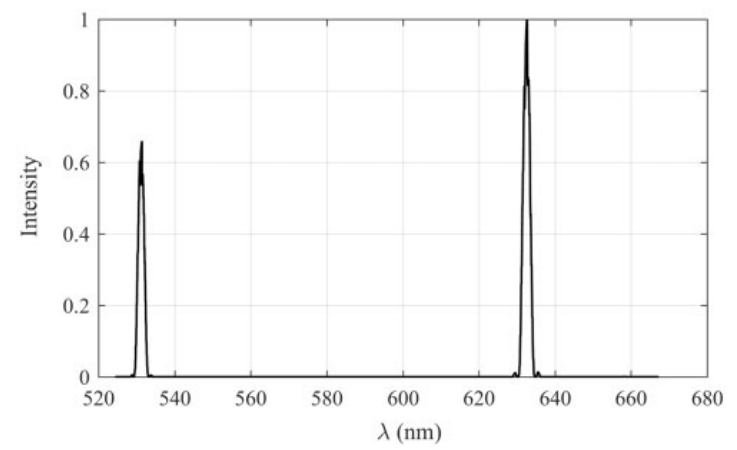

Figure 7. The recorded spectrum of one green $(532 \mathrm{~nm})$ and one red $(632.8 \mathrm{~nm}) \mathrm{He}-\mathrm{Ne}$ lasers by the static-FTS. The measured bandwidths are roughly $1.9 \mathrm{~nm}$ and $2.1 \mathrm{~nm}$ for the green and the red $\mathrm{HeNe}$ laser, respectively.

have been $0.11 \mathrm{~nm}$ or $3.5 \mathrm{~cm}^{-1}$. While using the static-FTS, the pixel resolution of the device depends on the inverse of maximum delay and it is roughly $0.05 \mathrm{~nm}$ or $1.9 \mathrm{~cm}^{-1}$. However, at the end, overall optical resolution will be determined by the pixel resolution times the dispersion of the system which depends on the optics in front of the interferometer, such as the pinhole size, the properties of the curved mirror, and the grating pair.

The device bandwidth and the resolution are limited by the CCD camera dimensions; in this work we used a 1.3 MP PixeLINK CCD camera with a CCD array size of $6.65 \times 5.32 \mathrm{~nm}$. However, the method is scalable such that increasing the CCD dimensions will increase the bandwidth and the resolution of the spectrometer. The minimum detectable power with the current set-up is about $50 \mu \mathrm{W}$ in $50 \mathrm{~ms}$ of recording time.

The proposed spectrometer is a combination of a staticFTS and dispersive spectrometer; therefore, it carries both advantages and disadvantages of either spectrometer.
The broadband advantage is due to the dispersive part, while the high-speed and high-resolution advantages come from the FTS part. Although all FTSs require some aperture to control the convergence of the beam in the interferometer, this is more of an issue here. In our static-FTS, we have to enlarge and collimate the beam in both dimensions; therefore, it has to go through a pinhole beforehand. Consequently, low throughput disadvantage appears. For a given pinhole size, throughput will depend on the bandwidth of the signal, since the narrow band signals can be focused in a smaller area.

Furthermore, this spectrometer has a mixture of a multiplex advantage within a segment and a multichannel advantage in that it collects segments simultaneously. Considering the shot noise of the CCD, calculations show that for broadband signals, traditional FTSs and our proposed FTS have a similar S/N ratio performance. ${ }^{18}$ However, for the narrowband signals, the traditional FTSs show $\sqrt{M}$ times better $\mathrm{S} / \mathrm{N}$ ratio performance than the static-FTS proposed. As far as the dispersive spectrometers with $C C D$ arrays are concerned, from the CCD point of view, the $S / N$ ratio performance of our spectrometer is the same as the dispersive spectrometers since each segment is detected independently.

\section{Conflict of Interest}

The authors report there are no conflicts of interest.

\section{Funding}

This project was funded by The Scientific and Technological Research Council of Turkey (TÜBITAK, project no. I I3F357).

\section{References}

I. B. Schrader. Infrared and Raman Spectroscopy: Methods and Applications. Weinheim: VCH, 1995.

2. M.J. Baker, J. Trevisan, P. Bassan, R. Bhargava, et al. "Using Fourier Transform IR Spectroscopy to Analyze Biological Materials". Nat. Protoc. 20|4. 9(8): |77|-|79|.

3. N. Prieto, O. Pawluczyk, M.E.R. Dugan, J.L. Aalhus. "A Review of the Principles and Applications of Near-Infrared Spectroscopy to Characterize Meat, Fat, and Meat Products". Appl. Spectrosc. 2017. $71(7):$ I403-1426.

4. S. Kazarian, K. Chan. "Applications of ATR-FTIR Spectroscopic Imaging to Biomedical Samples". Biochim. Biophys. Acta. 2006. 1758(7): 858-867.

5. Y. Chen, C. Zou, M. Mastalerz, S. Hu, et al. "Applications of MicroFourier Transform Infrared Spectroscopy (FTIR) in the Geological Sciences A Review". Int. J. Mol. Sci. 20I5. 16(I2): 30223-30250.

6. B.C. Smith. Fundamentals of Fourier Transform Infrared Spectroscopy. Boca Raton, FL: CRC Press, $201 \mathrm{I}$.

7. V. Saptari. Fourier Transform Spectroscopy Instrumentation Engineering. Bellingham Washington, DC: SPIE Optical Engineering Press, 2003.

8. P.R. Griffiths, J.A. De Haseth. Fourier Transform Infrared Spectrometry, Volume 17I. Hoboken, NJ: John Wiley and Sons, 2007.

9. A. Lacan, F.M. Bréon, A. Rosak, F. Brachet, et al. "A Static Fourier Transform Spectrometer for Atmospheric Sounding: Concept and Experimental Implementation”. Opt. Exp. 2010. 18(8): 83II-833I. 
10. K. Möller. "Wave-Front-Dividing Array Interferometers without Moving Parts for Real-Time Spectroscopy from the IR to the UV". Appl. Opt. 1995. 34(9): 1493-150I.

II. M.L. Junttila, J. Kauppinen, E. Ikonen. "Performance Limits of Stationary Fourier Spectrometers". JOSA A. 199I. 8(9): |457-| 462.

12. F.M. Reininger. "The Application of Large Format, Broadband Quantum Well Infrared Photodetector Arrays to Spatially Modulated Prism Interferometers". Infrared Phys. Technol. 2001. 42(3): 345-362.

13. J. Connes, P. Connes. "Near-Infrared Planetary Spectra by Fourier Spectroscopy. I. Instruments and Results". JOSA. 1966. 56(7): 896-910.

14. M. Schardt, P.J. Murr, M.S. Rauscher, A.J. Tremmel, et al. "Static Fourier Transform Infrared Spectrometer”. Opt. Exp. 2016. 24(7): 7767-7776.
15. B. Sardari, F. Davoli, M. Özcan. "A Broadband Configuration for Static Fourier Transform Spectroscopy with Bandpass Sampling". Rev. Sci. Inst. 2016. 87(10): 103106.

16. B. Sardari, F. Davoli, M. Özcan. "A Simple Configuration for Static Fourier Transform Infrared Spectrometers". In: F. Berghmans, A.G. Mignani. Optical Sensing and Detection IV. Proc. SPIE. 20I6. 9899: 98990T.

17. R.G. Vaughan, N.L. Scott, D.R. White. "The Theory of Bandpass Sampling”. IEEE Trans. Signal Process. 199I. 39(9): 1973-1984.

18. B. Sardari. Development of a Static Fourier Transform Spectrometer and Real-Time Substrates for Surface Enhanced Raman Scattering. [Ph.D. Thesis]. Istanbul, Turkey: Sabanci University, 2017. 